\title{
The existence of equilibrium in a simple exchange model
}

Piotr Maćkowiak*

\section{"Correspondence:}

p.mackowiak@ue.poznan.pl

Department of Mathematical

Economics, Poznań University of

Economics, Al. Niepodległości 10

Poznań, 61-875, Poland

\section{囪 Springer}

\begin{abstract}
This paper gives a new proof of the existence of equilibrium in a simple model of an exchange economy. We first formulate and prove a simple combinatorial lemma and then we use it to prove the existence of equilibrium. The combinatorial lemma allows us to derive an algorithm for the computation of equilibria. Though the existence theorem is formulated for functions defined on open simplices, it is equivalent to the Brouwer fixed point theorem.

MSC: Primary 91B02; secondary 91B50; 54H25
\end{abstract}

Keywords: simple exchange model; equilibrium existence; zero of a function; fixed point; computation of equilibria; simplicial methods

\section{Introduction}

Consider an economy with $n$ goods populated with a finite number $m$ of consumers whose preferences $\succeq_{i}$, defined on $\mathbb{R}_{+}^{n}$, are continuous, strictly monotone and strictly convex. ${ }^{a}$ Suppose also that each consumer possesses a stock $\omega^{i} \in \mathbb{R}_{+}^{n}$ of goods and that the (total) supply $\omega=\omega^{1}+\cdots+\omega^{m}$ is positive, $\omega>0$. Suppose that at each positive price vector $p=\left(p_{1}, \ldots, p_{n}\right)$ each consumer $i$ wants to maximize his/her preferences among affordable bundles of goods, i.e., he/she plans to buy a bundle of goods $x^{i}(p) \in \mathbb{R}^{n}$ such that its value $p x^{i}(p)$ is not greater than the value $p \omega^{i}$ of the disposable stock $\omega^{i}$ and $x^{i}(p)$ is the best among affordable bundles: $p x \leq p \omega^{i}, x \in \mathbb{R}_{+}^{n}, x \neq x^{i}(p)$ implies $x^{i}(p) \succeq_{i} x$ and it is not true that $x \geq_{i} x^{i}(p)$. The monotonicity of preferences implies that $p x^{i}(p)=p \omega^{i}$. Hence, at the given prices $p$, it holds $p x(p)=p \omega$, where $x(p)=x^{1}(p)+\cdots+x^{n}(p)$ is the (total) demand for goods at prices $p$. Plans of all consumers can come into effect only if $x(p)=\omega$ - again by the monotonicity assumption on preferences. Does there exist an equilibrium price vector, i.e., a positive price vector $p$ such that $x(p)=\omega$ ? It is well known that the answer to that question is positive; see [1] for a survey of the basic existence results. It is obvious that $p$ is an equilibrium price vector if and only if the difference $z(p):=x(p)-\omega$ vanishes. If we allow $p$ to vary over the positive orthant of $\mathbb{R}^{n}$, we obtain the function $z$; the excess demand function of the economy. One can show that $z$ is homogeneous of degree zero, continuous on the set of positive prices, it satisfies Walras' law and a boundary condition, and it is bounded from below [2, Theorem 1.4.4]. One can also show that if a function $f$ defined on the positive orthant of $\mathbb{R}^{n}$ possesses the properties listed in the previous sentence, then there exists an economy whose excess demand function $z$ is different from $f$ only on a neighborhood of the boundary of $\mathbb{R}_{+}^{n}$ in $\mathbb{R}^{n}$ and the set of equilibrium prices

(c) 2013 Maćkowiak: licensee Springer. This is an Open Access article distributed under the terms of the Creative Commons Attribution License (http://creativecommons.org/licenses/by/2.0), which permits unrestricted use, distribution, and reproduction in any medium, provided the original work is properly cited. 
for $z$ coincides with the set of zeros of $f$ [3]. In this work, we are going to use the excess demand approach to prove the existence of equilibrium [1, Section 3]: we just impose conditions a function should possess to be the excess demand function of an economy and then we prove that there exists an equilibrium price vector. ${ }^{\mathrm{b}}$ The novelty of our approach is that we are proving the existence of equilibrium (see the theorem in Section 4) in a new and constructive way. ${ }^{\mathrm{c}}$ It is important to emphasize that we do not rely on the Sperner lemma [4, p.19] to prove the result. Instead of that, we introduce a combinatorial lemma (Lemma 1) formulated for a special triangulation of a closed simplex only. The particular triangulation decreases generality of the lemma but is computationally advantageous $[4$, p.65]. ${ }^{\mathrm{d}}$

In the next section, we introduce notation. Section 3 presents necessary notions from combinatorial topology and ends with the combinatorial lemma (Lemma 1). In Section 4, we define the notions of excess demand function and equilibrium, and then we derive some properties of excess demand functions. Finally, we prove the existence theorem. Section 5 contains an algorithm for computation of equilibria. In Section 6, we clarify some differences between the boundary condition we use (see Definition 1(3)) and the standard boundary condition met in the literature. We also present a connection between fixed points of continuous functions and equilibria (zeros) of excess demand functions. At the end of Section 6, we pose a few open questions.

\section{Notation}

Let $\mathbb{N}$ denote the set of positive integers and for any $n \in \mathbb{N}$ let $\mathbb{R}^{n}$ denote the $n$ dimensional Euclidean space, and $[n]:=\{1, \ldots, n\},[0]:=\emptyset$. Moreover, $e^{i}$ is the $i$ th unit vector of the standard basis of $\mathbb{R}^{n}$, where $i \in[n]$. In what follows, for $n \in \mathbb{N}$ the set $\Delta^{n}:=\left\{x \in \mathbb{R}_{+}^{n}: \sum_{i=1}^{n} x_{i}=1\right\}$, where $\mathbb{R}_{+}$is the set of nonnegative real numbers, is the standard (n-1)-dimensional (closed) simplex and int $\Delta^{n}:=\left\{x \in \Delta^{n}: x_{i}>0, i \in[n]\right\}$ is its (relative) interior. For a set $X \subset \mathbb{R}^{n}, \partial(X)$ denotes its boundary (or relative boundary of the closure of $X$ if $X$ is convex). For vectors $x, y \in \mathbb{R}^{n}$ their scalar product is $x y=\sum_{i=1}^{n} x_{i} y_{i}$. The Euclidean norm of $x \in \mathbb{R}^{n}$ is denoted by $|x|$. For any set $A$, \#A denotes its cardinality.

\section{Definitions, facts and a combinatorial lemma}

We need some more or less standard definitions and facts from combinatorial topology; they can be found in [4] and [5]. Let us fix $n \in \mathbb{N}$.

- Let $v^{j} \in \mathbb{R}^{n}, j \in[k], k \leq n+1$, be affinely independent. The set $\sigma$ defined as $\sigma:=\left\{x \in \mathbb{R}^{n}: x=\sum_{j=1}^{k} \alpha_{j} v^{j}, \alpha \in \Delta^{k}\right\}$ is called a $(k-1)$-simplex with vertices $v^{j}, j \in[k]$. We write it briefly as $\sigma=\left\langle v^{j}: j \in[k]\right\rangle$ or $\sigma=\left\langle v^{1}, \ldots, v^{k}\right\rangle$. If we know that $\sigma$ is a $(k-1)$-simplex, then the set of its vertices is denoted by $V(\sigma)$. If $p \in \sigma$, then the vector $\alpha^{p}:=\left(\alpha_{1}^{p}, \ldots, \alpha_{k}^{p}\right) \in \Delta^{k}$ is called the vector of the barycentric coordinates of $p$ in $\sigma$, if $p=\sum_{j=1}^{k} \alpha_{j}^{p} v^{j}$. For each $p \in \sigma$, its barycentric coordinates $\alpha^{p}$ in the simplex $\sigma$ are uniquely determined.

- If $\sigma$ is a $(k-1)$-simplex, then $\langle A\rangle$, where $\emptyset \neq A \subset V(\sigma)$, is called a (\#A-1)-face of $\sigma$.

- A collection $\left\{\sigma_{j}: j \in[J]\right\}, J \in \mathbb{N}$, of nonempty subsets of a $(k-1)$-simplex $S \subset \mathbb{R}^{n}$, $0<k \leq n+1$, is called a triangulation of $S$ if it meets the following conditions:

1. $\sigma_{j}$ is a $(k-1)$-simplex, $j \in[J]$,

2. if $\sigma_{j} \cap \sigma_{j^{\prime}} \neq \emptyset$ for $j, j^{\prime} \in[J]$, then $\sigma_{j} \cap \sigma_{j^{\prime}}$ is a common face of $\sigma_{j}$ and $\sigma_{j^{\prime}}$,

3. $S=\bigcup_{j \in[]]} \sigma_{j}$. 
- Two different $(k-1)$-simplices $\sigma_{j}, \sigma_{j^{\prime}}, j, j^{\prime} \in[J], j \neq j^{\prime}$, in a triangulation of a $(k-1)$-simplex $S$ are adjacent if $\left\langle V(\sigma) \cap V\left(\sigma^{\prime}\right)\right\rangle$ is a $(k-2)$-face for both of them. Each $(k-2)$-face of a simplex $\sigma_{j}, j \in[J]$, is a $(k-2)$-face for exactly two different simplices in the triangulation, provided the $(k-2)$-face is not contained in $\partial(S)$.

- The $K$-triangulation of an $(n-1)$-simplex $S=\left\langle v^{1}, \ldots, v^{n}\right\rangle \subset \mathbb{R}^{n}$ with grid size $m^{-1}$, where $m$ is a positive integer, ${ }^{\mathrm{e}}$ is the collection of all $(n-1)$-simplices $\sigma$ of the form $\sigma=\left\langle p^{1}, p^{2}, \ldots, p^{n}\right\rangle$, where vertices $p^{1}, p^{2}, \ldots, p^{n} \in S$ satisfy the following conditions:

1. each barycentric coordinate $\alpha_{i}^{p^{1}}, i \in[n]$, of $p^{1}$ in $S$ is a nonnegative multiple of $m^{-1}$

2. $\alpha^{p^{j+1}}=\alpha^{p^{j}}+m^{-1}\left(e^{\pi_{j}}-e^{\pi_{j}+1}\right)$, where $\pi=\left(\pi_{1}, \ldots, \pi_{n-1}\right)$ is a permutation of $[n-1]$, $\alpha^{p^{l}}$ is the vector of the barycentric coordinates of $p^{l}, l \in\{j, j+1\}, j \in[n-1]$.

The $K$-triangulation of $S$ with grid size $m^{-1}$ is denoted by $K(S, m)$ and the set of all vertices of simplices in $K(S, m)$ is denoted by $V(S, m)$. Obviously, $V(S, m)=\bigcup_{\sigma \in K(S, m)} V(\sigma)=\left\{\alpha_{1} v^{1}+\cdots+\alpha_{n} v^{n}: \alpha \in \Delta^{n}, \alpha_{i} \in\{0,1 / m, \ldots, 1-1 / m, 1\}\right\}$. For any $\varepsilon>0$ and for a sufficiently large $m$, each simplex in $K(S, m)$ has the diameter not greater than $\varepsilon$. Moreover, there exists exactly one simplex in $K(S, m)$ such that $v^{n}$ is its vertex. ${ }^{\mathrm{f}}$

A basic tool used in the proof of our main result is the following.

Lemma 1 Let $S:=\left\langle v^{1}, \ldots, v^{n}\right\rangle \subset \mathbb{R}^{n}$ be an $(n-1)$-simplex and $l: V(S, m) \rightarrow\{0,1, \ldots, n\}$, $m \geq 2$, be a function satisfying for all $p \in V(S, m)$ the following conditions:

1. $\alpha_{i}^{p}=0 \Rightarrow l(p) \neq i, i \in[n-1]$,

2. $l(p)=0$ if $\alpha_{n}^{p}=0$,

3. $l(p)=n$ if $\alpha_{n}^{p}=1$,

4. $l(p) \in[n-1]$ if $0<\alpha_{n}^{p}<1$.

Then there exists a unique finite sequence of simplices $\sigma_{1}, \ldots, \sigma_{J} \in K(S, m), J \in \mathbb{N}$, such that $\sigma_{j}$ and $\sigma_{j+1}$ are adjacent for $j \in[J-1], n \in l\left(\sigma_{1}\right), 0 \in l\left(\sigma_{J}\right),[n-1] \subset l\left(\sigma_{j}\right), j \in[J]$, and $\sigma_{j+1} \notin$ $\left\{\sigma_{1}, \ldots, \sigma_{j}\right\}, j \in[J-1] .{ }^{g}$

Proof Let $\sigma_{1}$ denote the unique simplex in $K(S, m)$ whose vertex is $p^{n}:=v^{n}$. Vectors of the barycentric coordinates of vertices of $\sigma_{1}$ (other than $p^{n}$ ) are of the form

$$
\alpha^{p^{j}}=(0, \ldots, 0, \underbrace{m^{-1}}_{j \text { th coordinate }}, 0, \ldots, 0,1-m^{-1}), \quad j \in[n-1] .
$$

Since $\alpha_{i}^{p^{j}}=0$ implies $l\left(p^{j}\right) \neq i$, then $l\left(p^{j}\right)=j, j \in[n]$ and therefore $[n-1] \subset l\left(\sigma_{1}\right)$. Moreover, since for all $v \in V(S, m) \alpha_{i}^{v}=0$ implies $l(v) \neq i$, then $l\left(\sigma^{\prime}\right)=[n-1]$ entails $\sigma^{\prime}$ is not contained in $\partial(S)$, where $\sigma^{\prime}$ is an $(n-2)$-face of some $\sigma \in K(S, m)$. Whence, no $(n-2)$ face of $\sigma \in K(S, m)$ on whose vertices function $l$ assumes all values in $[n-1]$ is contained in the boundary of $S$. Further, there exists exactly one $\sigma_{2} \in K(S, m) \backslash\left\{\sigma_{1}\right\}$, which is adjacent to $\sigma_{1}$. Obviously, $l\left(\sigma_{2}\right)=[n-1]$. Let $p_{n+1}$ be the only element of $V\left(\sigma_{2}\right) \backslash V\left(\sigma_{1}\right)$. Since $l\left(\left\{p^{1}, \ldots, p^{n-1}\right\}\right)=[n-1]$ and $l\left(p^{n+1}\right) \in[n-1]$, there exists exactly one vertex $p^{i_{1}}$ among $p^{1}, \ldots, p^{n-1}$ such that $l\left(p^{i_{1}}\right)=l\left(p^{n+1}\right)$ and function $l$ attains all values in $[n-1]$ on the $(n-2)$ face $\left\langle V\left(\sigma^{2}\right) \backslash\left\{p^{i_{1}}\right\}\right\rangle$. So, we can find a simplex $\sigma_{3} \in K(S, m) \backslash\left\{\sigma_{1}, \sigma_{2}\right\}$ adjacent to $\sigma_{2}$ with $[n-1] \subset l\left(\sigma_{3}\right)$, and if $0 \in l\left(\sigma_{3}\right)$ - the process is complete, if not - proceeding as earlier we can find a simplex $\sigma_{4} \in K(S, m) \backslash\left\{\sigma_{1}, \sigma_{2}, \sigma_{3}\right\}$ and so on. ${ }^{\text {h }}$ Suppose we have constructed 


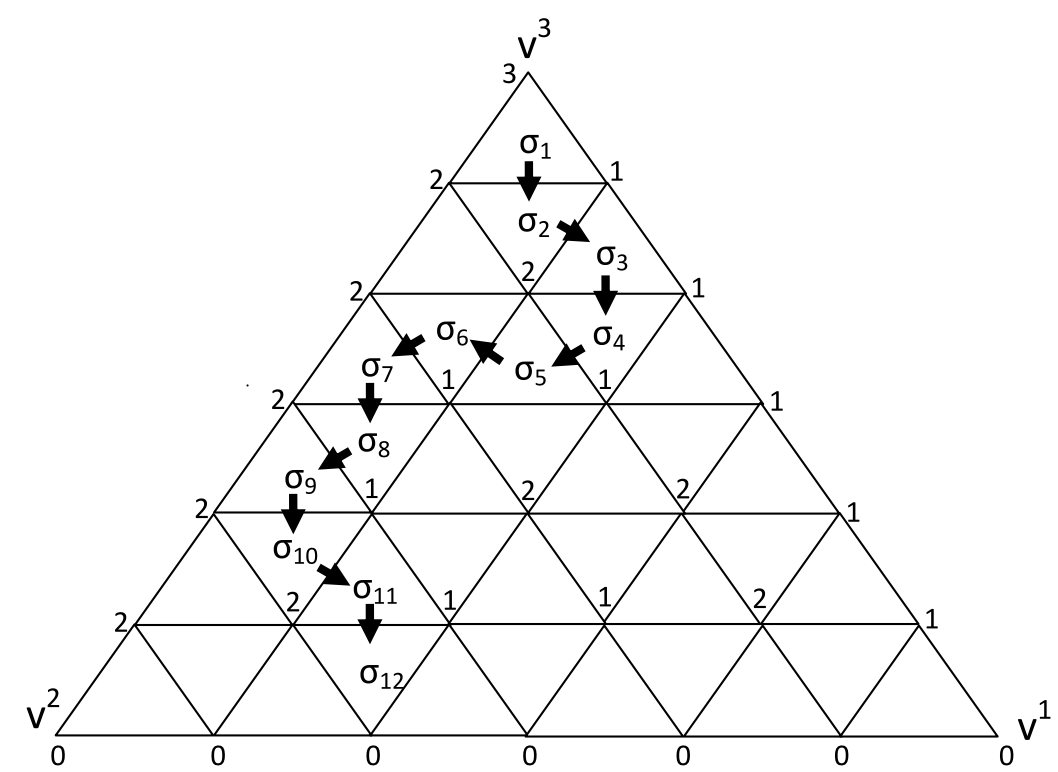

Figure 1 The $K$-triangulation of 2 -simplex $S=\left\langle v^{1}, v^{2}, v^{3}\right\rangle$ with grid size 6 . The small triangles are members of the triangulation $K(S, 6)$. The number at a vertex of a simplex in $K(S, 6)$ is the value of $/$ assigned to the vertex and one sees that / satisfies the assumptions of Lemma 1. The sequence of simplices $\sigma_{1}, \ldots, \sigma_{12}$ meets the requirements described in the proof of Lemma 1.

the sequence $\sigma_{1}, \ldots, \sigma_{J}$. If $0 \in l\left(\sigma_{J}\right)$, then the sequence satisfies the claim. Suppose that $0 \notin l\left(\sigma_{J}\right)$. Since each $(n-2)$-face which is not contained in $\partial(S)$ is shared by exactly two simplices of $K(S, m)$, there exists precisely one simplex $\sigma^{\prime}$ in $K(S, m) \backslash\left\{\sigma_{1}, \ldots, \sigma_{J}\right\}$ such that $\sigma_{J}$ and $\sigma^{\prime}$ share the $(n-2)$-face $\sigma^{\prime} \cap \sigma_{J}$ with $l\left(\sigma^{\prime} \cap \sigma_{J}\right)=[n-1]$ - this ensures that $\sigma_{J+1}=\sigma^{\prime}$ and that no simplex of $K(S, m)$ appears twice (or more) in the sequence $\sigma_{1}, \ldots, \sigma_{J+1}$, where $0 \notin l\left(\sigma_{J}\right)$. Thus, in view of the finiteness of $K(S, m)$ and since $l\left(\sigma^{\prime}\right)=[n-1]$ implies $\sigma^{\prime}$ is not contained in $\partial(S)$, we conclude that there exists $J$ such that $0 \in l\left(\sigma_{J}\right)$, otherwise we could construct an infinite sequence of simplices built of finitely many different elements of $K(S, m)$, which would imply that a simplex appears more than once in the sequence which is an absurd. The choice of $\sigma_{j+1}$ guarantees that $\sigma_{j+1} \notin\left\{\sigma_{1}, \ldots, \sigma_{j}\right\}, j \in[J-1]$. Uniqueness of the constructed sequence comes from the preceding sentence, uniqueness of the simplex containing $v^{n}$, and the fact that each $(n-2)$-face in the (relative) interior of $S$ is shared by exactly two simplices of the triangulation.

\section{The existence of equilibrium}

Definition 1 Let us fix $n \in \mathbb{N}$. We say that a function $z$ : int $\Delta^{n} \rightarrow \mathbb{R}^{n}, z(p)=\left(z_{1}(p), \ldots\right.$, $z_{n}(p)$ ), is an excess demand function, if it satisfies the following conditions:

1. $z$ is continuous on int $\Delta^{n}$,

2. Walras' law holds, that is, $p z(p)=0$ for $p \in \operatorname{int} \Delta^{n}$,

3. the boundary condition holds: if $p^{j} \in$ int $\Delta^{n}, j \in \mathbb{N}, \lim _{j \rightarrow+\infty} p^{j}=p \in \partial\left(\Delta^{n}\right)$ and $p_{i}=0, i \in[n]$, then $\lim _{j \rightarrow+\infty} z_{i}\left(p^{j}\right)=+\infty$,

4. $z$ is bounded from below: $\inf _{p \in \text { int } \Delta^{n}} z_{i}(p)>-\infty, i \in[n]$.

Definition 2 Let $z$ : int $\Delta^{n} \rightarrow \mathbb{R}^{n}$ be an excess demand function, $n \in \mathbb{N}$. A point $p \in$ int $\Delta^{n}$ is called an equilibrium point for $z$, if $z(p)=0$. 
The main goal of the paper is to give a new proof of the fact that for each excess demand function there exists an equilibrium point. First, we are going to characterize the behavior of $z$ near the (relative) boundary of its domain, which is crucial for the theorem to follow. The intuition for the lemma below is as follows: if the price $p_{i}$ of a good $i$ is low (in comparison to some other price - prices are standardized; they sum up to 1) then the demand significantly exceeds the supply of that good; if the price $p_{i}$ is (relatively) high - so all the other prices are low - then the demand for the $i$ th good is considerably less than its supply.

Lemma 2 Let $z$ : int $\Delta^{n} \rightarrow \mathbb{R}^{n}$ be an excess demand function. Then there exists $\varepsilon_{1}>0$ such that for $i \in[n]$ and $p \in \operatorname{int} \Delta^{n}$ we have

$$
\left(p_{i} \leq \varepsilon_{1} \Rightarrow z_{i}(p)>0\right) \quad \text { and } \quad\left(p_{i} \geq 1-\varepsilon_{1} \Rightarrow z_{i}(p)<0\right) .
$$

Proof Suppose that the former implication is not true. Then there exist $i \in[n]$ and a sequence $p^{j} \in$ int $\Delta^{n}, j \in \mathbb{N}: \lim _{j \rightarrow+\infty} p^{j}=p, p_{i}=0$, and $\lim _{j \rightarrow+\infty} z_{i}\left(p^{j}\right) \leq 0$, which contradicts the boundary condition. This implies that there exists $\varepsilon_{1}>0$ for which the just considered implication is true and without loss of generality we can assume that $\varepsilon_{1}<1-\varepsilon_{1}$. To prove the latter implication, observe that $p_{i} \geq 1-\varepsilon_{1}$ implies $p_{i^{\prime}} \leq \varepsilon_{1}, i \neq i^{\prime}$, so the first implication guarantees that $z_{i^{\prime}}(p)>0, i^{\prime} \neq i$. Now, from Walras' law, we get $0<\sum_{i^{\prime} \neq i} p_{i^{\prime}} z_{i^{\prime}}(p)=-p_{i} z_{i}(p)$, and $z_{i}(p)<0$ is satisfied.

Lemma 3 Let $z$ and $\varepsilon_{1}$ be as in Lemma 2 . Let $S_{1}:=\left\{p \in\right.$ int $\left.\Delta^{n}: p_{n} \in\left(0,1-\varepsilon_{1} / 2\right]\right\}$ and define the function $\tilde{z}:$ int $\Delta^{n} \rightarrow \mathbb{R}^{n-1}$ as follows:

$$
\forall p \in \operatorname{int} \Delta^{n} \quad \widetilde{z}(p):=\left(\left(1-p_{n}\right) z_{i}(p)+p_{n} z_{n}(p)\right)_{i=1}^{n-1} .
$$

Then

1. $\tilde{z}$ is continuous,

2. $\tilde{z}$ is bounded from below: $\inf _{p \in \operatorname{int} \Delta^{n}} \widetilde{z}_{i}(p)>-\infty, i \in[n-1]$,

3. $p_{1} \widetilde{z}_{1}(p)+\cdots+p_{n-1} \widetilde{z}_{n-1}(p)=0$ for $p \in$ int $\Delta^{n}$,

4. if $p^{j} \in S_{1}, j \in \mathbb{N}, \lim _{j \rightarrow+\infty} p^{j}=p \in \partial\left(\Delta^{n}\right)$ and $p_{i}=0, i \in[n-1]$, then $\lim _{j \rightarrow+\infty} \widetilde{z}_{i}\left(p^{j}\right)=+\infty$,

5. $\exists \varepsilon_{2} \in\left(0, \varepsilon_{1} / 2\right] \forall p \in S_{1} \forall i \in[n-1]$ :

$$
\left(p_{i} \leq \varepsilon_{2} \Rightarrow \widetilde{z}_{i}(p)>0\right) \text { and }\left(p_{i} \geq 1-\varepsilon_{2} \Rightarrow \widetilde{z}_{i}(p)<0\right) .
$$

Proof The continuity of $\widetilde{z}$ is obvious. The boundedness from below of $\widetilde{z}$ stems from the fact that $z$ is bounded from below and the weights $p_{n}, 1-p_{n}$, are positive and less than 1 for all $p_{n} \in(0,1)$. The following equalities show that property (3) is met:

$$
\begin{aligned}
p_{1} & \widetilde{z}_{1}(p)+\cdots+p_{n-1} \widetilde{z}_{n-1}(p) \\
& =p_{1}\left(\left(1-p_{n}\right) z_{1}(p)+p_{n} z_{n}(p)\right)+\cdots+p_{n-1}\left(\left(1-p_{n}\right) z_{n-1}(p)+p_{n} z_{n}(p)\right) \\
& =\left(1-p_{n}\right)\left(p_{1} z_{1}(p)+\cdots+p_{n-1} z_{n-1}(p)\right)+\underbrace{\left(p_{1}+\cdots+p_{n-1}\right)}_{=1-p_{n}} p_{n} z_{n}(p) \\
& =\left(1-p_{n}\right) p z(p)=0 .
\end{aligned}
$$


If $p^{j} \in S_{1}, j \in \mathbb{N}$, converges to a point $p$ with $p_{i}=0$ for some $i \in[n-1]$ then $(1-$ $\left.p_{n}^{j}\right) z_{i}\left(p^{j}\right)$ diverges to $+\infty$ and since the product $p_{n}^{j} z_{n}\left(p^{j}\right)$ is bounded from below it holds: $\lim _{j \rightarrow+\infty} \widetilde{z}_{i}\left(p^{j}\right)=+\infty$. To prove that (5) is true it suffices to observe that for $p \in S_{1}$ we have $1-p_{n} \geq \varepsilon_{1} / 2$ and to proceed as in the proof of Lemma 2 with $\widetilde{z}$ in place of $z$.

The formula used to define the function $\widetilde{z}$ resembles the linear homotopy between functions

$$
\left(\left(1-\varepsilon_{1} / 2\right) z_{1}\left(\cdot, \varepsilon_{1} / 2\right)+\left(\varepsilon_{1} / 2\right) z_{n}\left(\cdot, \varepsilon_{1} / 2\right)\right)_{i=1}^{n-1}
$$

and

$$
\left(\left(\varepsilon_{1} / 2\right) z_{1}\left(\cdot, 1-\varepsilon_{1} / 2\right)+\left(1-\varepsilon_{1} / 2\right) z_{n}\left(\cdot, 1-\varepsilon_{1} / 2\right)\right)_{i=1}^{n-1} ;
$$

just put $t$ in place of $p_{n}$, assume that $t$ changes from $\varepsilon_{1} / 2$ through $1-\varepsilon_{1} / 2$ and the "homotopy' is

$$
H\left(p_{1}, \ldots, p_{n-1}, t\right):=\left((1-t) z_{i}(\cdot, t)+t z_{n}(\cdot, t)\right)_{i=1}^{n-1} .
$$

But $H$ is not a homotopy since the domain of $H(\cdot, t)$ changes as $t$ changes.

The important thing which Lemma 3 reveals is that at each fixed $p_{n} \in(0,1)$ the function $\widetilde{z}\left(\cdot, p_{n}\right)$ is an excess demand function defined on a simplex of dimension $n-2$ instead of $n-1$. $^{\mathrm{i}}$

Now suppose that $\varepsilon_{1}$ and $\varepsilon_{2}$ satisfy the statement of Lemma 3 and let for $i \in[n]$ :

$$
\bar{e}^{i}:=(\frac{\varepsilon_{2}}{n-1}, \ldots, \frac{\varepsilon_{2}}{n-1}, \underbrace{1-\varepsilon_{2}}_{i \text { th coordinate }}, \frac{\varepsilon_{2}}{n-1}, \ldots, \frac{\varepsilon_{2}}{n-1}) \in \operatorname{int} \Delta^{n} .
$$

We can assume that the vectors $\bar{e}^{i}, i \in[n]$, are linearly independent; it suffices to take sufficiently small $\varepsilon_{2}>0$. The set $S_{2}:=\left\langle\bar{e}^{i}: i \in[n]\right\rangle \subset$ int $\Delta^{n}$ is an $(n-1)$-simplex with the vertices $\bar{e}^{i}, i \in[n]$. If $p \in S_{2} \cap S_{1}$, then $p_{i} \in\left[\varepsilon_{2} /(n-1), 1-\varepsilon_{2}\right], i \in[n-1]$ and if $\alpha_{i}^{p}=0$ (i.e., $\left.p_{i}=\varepsilon_{2} /(n-1)<\varepsilon_{1} / 2\right)$ then $\widetilde{z}_{i}(p)>0$; similarly, if $\alpha_{i}^{p}=1$ (i.e. $\left.p_{i}=1-\varepsilon_{2}>1-\varepsilon_{1} / 2\right)$ then $\widetilde{z}_{i}(p)<0$. Moreover, if $p \in S_{2}$ and $p_{n} \geq 1-\varepsilon_{1}$ then $z_{n}(p)<0$ and if $p_{n} \leq \varepsilon_{1}$ then $z_{n}(p)>0$ (see Lemma 2). We are now in a position to prove the main result of the paper.

Theorem Let $z$ be as in Lemma 3. For each $\varepsilon>0$ there exists $p \in \operatorname{int} \Delta^{n}: z_{i}(p) \leq \varepsilon, i \in[n]$.

Proof If $n=1$, then there is nothing to prove: int $\Delta^{1}=\{1\} \subset \mathbb{R}$, and by Walras' law, $z(p)=0$ at $p=1$. Suppose that $n \geq 2$. Let us fix $\varepsilon>0$ and define $\varepsilon^{\prime}:=\varepsilon \varepsilon_{1}$, where $\varepsilon_{1}$ comes from Lemma 2. Let also $S_{1}$ be as in the hypothesis of Lemma 3 and let $S_{2}$ be the $(n-1)$-simplex with vertices given by (2). By the continuity of the restriction of $\widetilde{z}$ to the compact set $S_{2}$, there exists $\delta>0$ such that if $p, p^{\prime} \in S_{2}$ and $\left|p-p^{\prime}\right|<\delta$, then $\left|\widetilde{z}(p)-\widetilde{z}\left(p^{\prime}\right)\right|<\varepsilon^{\prime}$. Choose an integer $m \geq 2$ for which all simplices in $K\left(S_{2}, m\right)$ have diameter less than $\min \left\{\delta, \varepsilon_{1} / 4\right\}$. Let $k_{1}$ denote the smallest integer in $[m]$ for which $\left(1-\frac{k_{1}}{m}\right) \frac{\varepsilon_{2}}{n-1}+\left(1-\varepsilon_{2}\right) \frac{k_{1}}{m} \geq 1-\frac{\varepsilon_{1}}{2}$ - this ensures that a point $p \in S_{2}$ whose last barycentric coordinate in $S_{2}$ is greater than or equal to $k_{1} / m$ 
satisfies $p_{n} \geq 1-\varepsilon_{1} / 2$. To justify this statement, observe that $1-\varepsilon_{2}-\frac{\varepsilon_{2}}{n-1} \geq 1-2 \varepsilon_{2} \geq 1-\varepsilon_{1}>0$ and $\alpha_{n}^{p} \geq k_{1} / m$ entail

$$
\begin{aligned}
p_{n} & =\left(1-\alpha_{n}^{p}\right) \frac{\varepsilon_{2}}{n-1}+\left(1-\varepsilon_{2}\right) \alpha_{n}^{p}=\frac{\varepsilon_{2}}{n-1}+\left(1-\varepsilon_{2}-\frac{\varepsilon_{2}}{n-1}\right) \alpha_{n}^{p} \\
& \geq \frac{\varepsilon_{2}}{n-1}+\left(1-\varepsilon_{2}-\frac{\varepsilon_{2}}{n-1}\right) \frac{k_{1}}{m}=\left(1-\frac{k_{1}}{m}\right) \frac{\varepsilon_{2}}{n-1}+\left(1-\varepsilon_{2}\right) \frac{k_{1}}{m} \\
& \geq 1-\varepsilon_{1} / 2 .
\end{aligned}
$$

The minimality of $k_{1}$ assures that for any nonnegative integer $k<k_{1}$ if $p \in S_{2}$ and $\alpha_{n}^{p} \leq k / m$, then $p_{n}<1-\varepsilon_{1} / 2$ and $p \in S_{1}$; the latter implies that the claim of Lemma 3(5) applies to $p$. Notice that if $p \in S_{2}$ and $p_{n} \geq 1-\varepsilon_{1} / 2$ then $z_{n}(p)<0$ and if $p_{n}<\varepsilon_{1} / 2$ then $z_{n}(p)>0$ (see Lemma 2). Let us define a function $l$ from the set of vertices $V\left(S_{2}, m\right)$ to $[n] \cup\{0\}$ as follows: ${ }^{j}$

$$
l(p)= \begin{cases}n, & \text { if } \alpha_{n}^{p}=1, \\ 0, & \text { if } \alpha_{n}^{p}=0, \\ \min \left\{i \in[n-1]: \alpha_{i}^{p}>0\right\}, & \text { if } 1>\alpha_{n}^{p} \geq k_{1} / m, \\ \min \left\{i \in[n-1]: \widetilde{z}_{i}(p) \leq 0\right\}, & \text { if } k_{1} / m>\alpha_{n}^{p}>0,\end{cases}
$$

where $\widetilde{z}$ is defined in (1). For $i \in[n-1]$, if $p \in V\left(S_{2}, m\right), 1>\alpha_{n}^{p} \geq k_{1} / m$, and $\alpha_{i}^{p}=0$ then it is clear that $l(p) \neq i$, since if $l(p)=i$, then we would obtain $\alpha_{i}^{p}>0$. Assume that $p \in V\left(S_{2}, m\right)$ and $0<\alpha_{n}^{p}<k_{1} / m$. Since $p \in \operatorname{int} \Delta^{n}$, Lemma 3(3) ensures that $\widetilde{z}_{i}(p) \leq 0$ for some $i \in[n-1]$ - so, $l(p)$ is well defined. Moreover, $\alpha_{n}^{p}<k_{1} / m$ implies $\alpha_{n}^{p}=k / m$ for some nonnegative integer $k$ such that $k<k_{1}$ and, therefore, due to Lemma 3(5), it holds that $\widetilde{z}_{i}(p)>0$ for $\alpha_{i}^{p}=0$ from which we obtain $l(p) \neq i$ whenever $\alpha_{i}^{p}=0$. Therefore, the assumptions of the combinatorial Lemma 1 are satisfied. Hence, there exists a sequence of simplices $\sigma_{1}, \ldots, \sigma_{J}$ in $K\left(S_{2}, m\right)$ such that $\sigma_{j}$ and $\sigma_{j+1}$ are adjacent and $n \in l\left(\sigma_{1}\right), 0 \in l\left(\sigma_{J}\right),[n-1] \subset l\left(\sigma_{j}\right), j \in[J]$. There exists the first simplex in that sequence, call it $\sigma_{j_{1}}$, such that for all $j>j_{1}$ the last barycentric coordinate of all vertices of $\sigma_{j}$ in $S_{2}$ are less than $k_{1} / m$. Simplices $\sigma_{j_{1}} \cap \sigma_{j_{1}+1}$ are adjacent, i.e. they share an $(n-2)$-face, and in other words, they differ by one vertex only. By the choice of $j_{1}$ all vertices $p \in V\left(\sigma_{j_{1}+1}\right)$ satisfy $\alpha_{n}^{p}<k_{1} / m$, and there is a vertex $\bar{p} \in V\left(\sigma_{j_{1}}\right) \backslash V\left(\sigma_{j_{1}+1}\right)$ such that $\alpha_{n}^{\bar{p}} \geq k_{1} / m$. Now, the adjacency of $\sigma_{j_{1}}$ and $\sigma_{j_{1}+1}$, the fact that all simplices in $K(S, m)$ have diameters less than $\varepsilon / 4$ and the inequality $\bar{p}_{n} \geq 1-\varepsilon_{1} / 2$ entail that $p_{n} \geq 1-\varepsilon_{1}$ for $p \in V\left(\sigma_{j_{1}+1}\right)$, which implies $z_{n}(p)<0$ for $p \in V\left(\sigma_{j_{1}+1}\right)$. Reasoning analogously, we get for the last simplex, $\sigma_{J}$, that it holds: $z_{n}(p)>0, p \in V\left(\sigma_{J}\right)$. By the choice of $j_{1}$, all simplices $\sigma_{j}, j \geq j_{1}+1$, are contained in $S_{1} \cap S_{2}$. Moreover, their diameters are less than $\delta$ so $p, p^{\prime} \in \sigma_{j}, j \geq j_{1}+1$, implies $\left|\widetilde{z}_{i}(p)-\widetilde{z}_{i}\left(p^{\prime}\right)\right| \leq \varepsilon^{\prime}, i \in[n-1]$. Since $\bigcup_{j \geq j_{1}} \sigma_{j}$ is (arcwise) connected and $V\left(\sigma_{j_{1}}\right) \cap z_{n}^{-1}((-\infty, 0)) \neq \emptyset$ and $V\left(\sigma_{J}\right) \cap z_{n}^{-1}((0,+\infty)) \neq \emptyset$ then by the continuity of $\widetilde{z}$ there exists a simplex $\sigma_{j_{2}}, j_{2} \geq j_{1}+1: 0 \in z_{n}\left(\sigma_{j_{2}}\right)$. Let $p \in \sigma_{j_{2}}$ : $z_{n}(p)=0$. So $\left|p-p^{\prime}\right|<\delta, p^{\prime} \in V\left(\sigma_{j_{2}}\right)$. Since for each $i$, there exists a vertex $p^{i}$ of $\sigma_{j_{2}}$ such that $\widetilde{z}_{i}\left(p^{i}\right) \leq 0$ (by the inclusion $\left.[n-1] \subset l\left(\sigma_{j_{2}}\right)\right),\left(1-p_{n}\right) z_{i}(p)=\widetilde{z}_{i}(p) \leq \widetilde{z}_{i}\left(p^{i}\right)+\varepsilon^{\prime} \leq \varepsilon^{\prime}$, $i \in[n-1]$. Further, $z_{i}(p) \leq \frac{\varepsilon^{\prime}}{\left(1-p_{n}\right)} \leq \frac{\varepsilon^{\prime}}{\varepsilon_{1}}=\varepsilon, i \in[n-1]$, since $p_{n} \in\left[\varepsilon_{1}, 1-\varepsilon_{1}\right]$, if $z_{n}(p)=0$, due to Lemma 2. We have found a point $p \in$ int $\Delta^{n}: z_{i}(p) \leq \varepsilon, i \in[n]$, which ends the proof. 


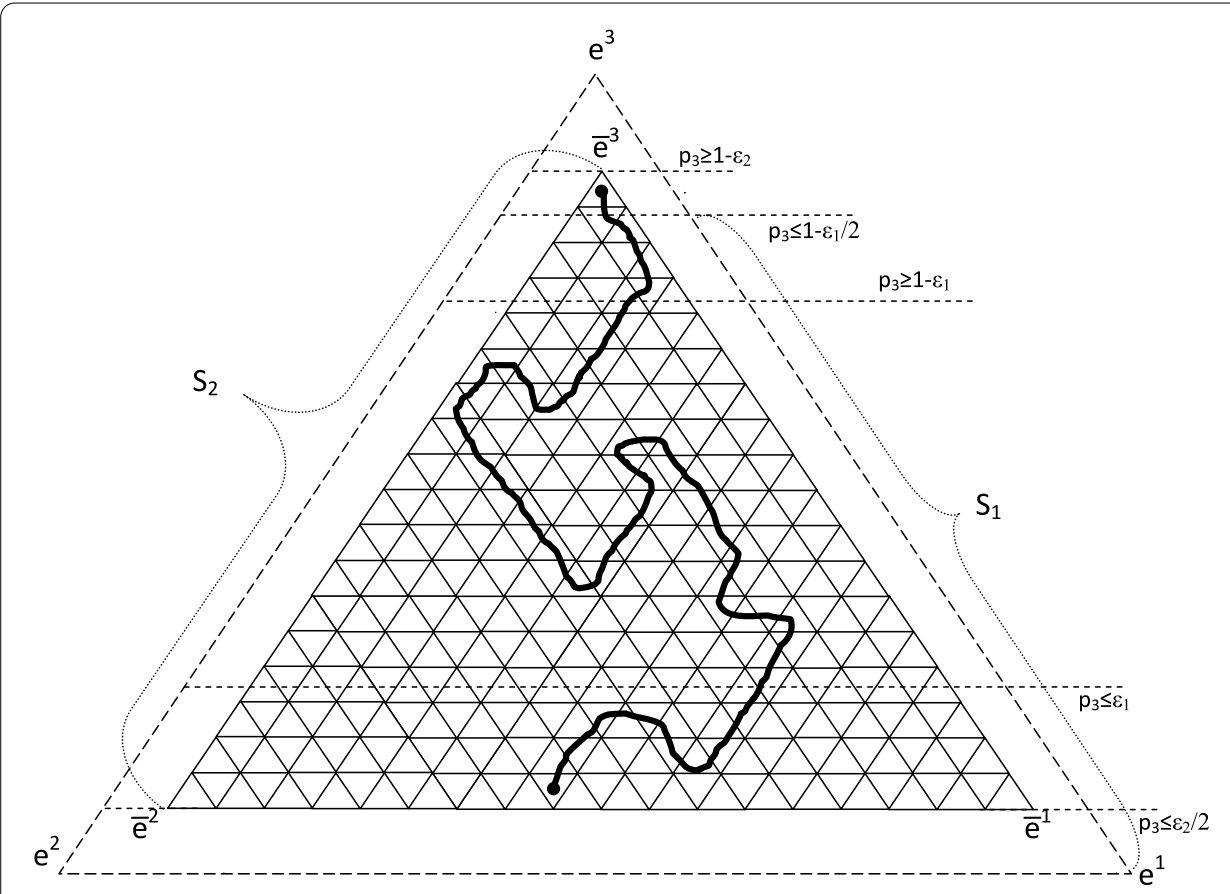

Figure 2 This figure explains the idea of the proof of theorem for $\boldsymbol{n}=\mathbf{3}$. Values of $/$ assigned to the vertices in $V\left(S_{2}, m\right)$ are independent of $\widetilde{z}$ if the considered vertex is above or on the line $p_{3} \leq 1-\varepsilon_{1} / 2$ - here the second and third row of the formula (3) are used to define vales of $I$. If a vertex is below the line $p_{3} \leq 1-\varepsilon_{1} / 2$ - but not at the bottom of $S_{2}$ - then $\tilde{z}$ is used to compute the value of $I$. The thick curve presents a hypothetical sequence of simplices $\sigma_{1}, \ldots, \sigma_{\jmath}$. For vertices of simplices above $\left(p_{3} \geq 1-\varepsilon_{1}\right)$-line (below $\left(p_{3} \leq \varepsilon_{1}\right)$-line) values of $z_{n}$ are negative (positive). If $\sigma_{j}$ is below $\left(p_{3} \leq 1-\varepsilon_{1} / 2\right)$-line then each coordinate of $\tilde{z}$ admits a non-positive value at a vertex of $\sigma_{j}$. Somewhere between $\left(p_{3} \geq 1-\varepsilon_{1}\right)$ and $\left(p_{3} \leq \varepsilon_{1}\right)$-lines there is a simplex $\sigma_{j}$ such that $z_{n}(p) z_{n}\left(p^{\prime}\right) \leq 0$ for a pair of vertices $p, p^{\prime}$ of $\sigma_{j}$ - that simplex is what we are looking for.

Figure 2 illustrates the proof.

Corollary Let $z$ be as in the above theorem. There exists an equilibrium point for $z$.

Proof Let $\varepsilon^{q}>0, q \in \mathbb{N}$, be a sequence converging to 0 . In view of the proof of the theorem, for each $q \in \mathbb{N}$ there exists a point $p^{q} \in S_{2}$ such that $z_{i}\left(p^{q}\right) \leq \varepsilon^{q}, i \in[n]$. The BolzanoWeierstrass theorem and compactness of $S_{2}$ imply that there exists a convergent subsequence $p^{q^{\prime}}$ of $p^{q}$, such that $\lim _{q^{\prime} \rightarrow+\infty} p^{q^{\prime}}=p \in S_{2}$. From the continuity of $z$, it follows that $z_{i}(p) \leq 0$, for $i \in[n]$. Since $p \in S_{2} \subset$ int $\Delta^{n}, p_{i}>0, i \in[n]$. Walras' law ensures that $z(p)=0$.

\section{An algorithm for the computation of equilibrium}

From the proof of the theorem, we can derive the following algorithm for computation of a point $p \in$ int $\Delta^{n}$ satisfying $z_{i}(p) \leq \varepsilon, i \in[n]$, where $\varepsilon>0$ is a given accuracy level. The algorithm below uses the function $l: V\left(S_{2}, m\right) \rightarrow\{0,1, \ldots, n\}$ defined in (3) and we reasonably assume that $n \geq 2$.

Step 0: Determine $\varepsilon_{1}, \varepsilon_{2}$ satisfying claim of Lemma 2 and Lemma 3(5), respectively. Fix accuracy level: $\varepsilon>0$. Find $\delta>0$ such that if $p, p^{\prime} \in S_{2}$, where $S_{2}$ is defined as in the proof of the theorem, and $\left|p-p^{\prime}\right|<\delta$ then $\left|\widetilde{z}(p)-\widetilde{z}\left(p^{\prime}\right)\right|<\varepsilon \varepsilon_{1}$ and let $m \geq 2$ be an 
integer for which all simplices in $K\left(S_{2}, m\right)$ have diameter less than $\min \left\{\delta, \varepsilon_{1} / 4\right\}$. Let $\sigma_{1}$ be is as in the proof of Lemma 1 for $S=S_{2}$, set FaceVertices $:=V\left(\sigma_{1}\right) \backslash\left\{\bar{e}^{n}\right\}, \bar{v}:=\bar{e}^{n}$ (see formula (2)) and go to step 1 .

Step 1: Determine the only vertex $v \in V\left(S_{2}, m\right)$ such that $v \neq \bar{v}$ and $\langle$ FaceVertices $\cup\{v\}\rangle \in K\left(S_{2}, m\right)$. Go to step 2 .

Step 2: If $\langle$ FaceVertices $\cup\{v\}\rangle \subset S_{1}$, where $S_{1}$ is defined in Lemma 3 , and $z_{n}(v)>0$ STOP: $v$ satisfies $z_{i}(v) \leq \varepsilon, i \in[n]$. Otherwise, assign the only element of $l^{-1}(l(v)) \cap$ FaceVertices as the value of $\bar{v}$. Set FaceVertices $:=($ FaceVertices $\backslash\{\bar{v}\}) \cup\{v\}$ and go to step 1 .

Step 0 initializes the necessary parameters for correct course of the algorithm and in fact it is the most difficult part of the algorithm, unless we know some properties of the considered excess demand function (e.g., differentiability, its lower bound or if it is a Lipschitz function on compact subsets of int $\left.\Delta^{n}\right)$. It is easy to determine $m$ if we know $\delta$ and $\varepsilon_{1}$; it suffices to take $m \geq \frac{(n-1) \sqrt{2}}{\min \left\{\delta, \varepsilon_{1} / 4\right\}}$, which is a consequence of the definition of the $K$-triangulation and the fact that the diameter of a simplex equals the maximum distance between its vertices. In Steps 1 and 2, set 〈FaceVertices〉 is a face of an element of $K\left(S_{2}, m\right)$ such that $l($ FaceVertices $)=[n-1]$. In Step 2, we check if currently considered simplex $\langle$ FaceVertices $\cup\{v\}\rangle$, where $v$ is such a vertex in $K\left(S_{2}, m\right)$ that $\langle$ FaceVertices $\rangle$ is common $(n-1)$-face of the currently considered simplex and its direct predecessor $\langle$ FaceVertices $\cup\{\bar{v}\}\rangle$, is contained in $S_{1}$, which implies that the value of $l$ depends on function $\widetilde{z}$ (see Lemma 3 and formula (3)). If it is the case, and in addition $z_{n}(v)>0$, then $v$ is what we seek for. If not, we have to find the next adjacent simplex; to this goal, we have to decide which vertex should be removed from FaceVertices. To achieve this, we find the vertex $\bar{v} \in$ FaceVertices, which bears the same value of $l$ as $v$ and we form the new set FaceVertices substituting $v$ in place of $\bar{v}$ and then we repeat the operations. The algorithm succeeds in finding approximate zero in a finite number of iterations due to Lemma 1 , the theorem and its proof. It is worth to emphasize that at a given iteration of the algorithm (Step 1-Step 2) exactly one new value of $l$ is computed and to proceed on with computations it is sufficient to know only the last simplex; there is no need to remember the earlier stages in the course of the algorithm. Moreover, the values of $l$ need to be computed only at the vertices of the constructed sequence of simplices.

\section{Final comments}

\subsection{The boundary condition}

The standard form of the boundary condition imposed on/satisfied by an excess demand functions is: ${ }^{\mathrm{k}} p^{j} \in \operatorname{int} \Delta^{n}, j \in \mathbb{N}, \lim _{j \rightarrow+\infty} p^{j}=p \in \partial\left(\Delta^{n}\right), p_{i}=0, \operatorname{implies}_{\lim _{j \rightarrow+\infty}} \max \left\{z_{i}\left(p^{j}\right)\right.$ : $i \in[n]\}=+\infty$. The difference is that we assume that if the (relative) price of a good $i$ tends to 0 , then the excess demand for the good $i$ goes to $+\infty$. The standard condition claims that if the (relative) price of a good $i$ tends to 0 , then the excess demand for some good, not necessarily $i$, goes to $+\infty$. Our condition is satisfied if there is a consumer with CobbDouglas preferences and owns a positive quantity of each good. But even if $z$ is an excess demand function that satisfies the standard boundary condition, we can approximate $z$ (as close as we wish on compact subsets of int $\Delta^{n}$ ) with an excess demand function satisfying the version of the boundary condition used in the paper; see the below construction of the function $z^{h}$ and just put there $z$ in place of $g$. 


\subsection{Fixed points of continuous functions defined on the standard simplex}

Here, we show how to relate a continuous function $f: \Delta^{n} \rightarrow \Delta^{n}$ and an excess demand function, for which we can apply our algorithm and we can find approximate fixed points of $f$. We use a construction by Uzawa [7]. Let a continuous function $g: \Delta^{n} \rightarrow \mathbb{R}^{n}$ be defined as

$$
\forall x \in \Delta^{n}: g(x):=f(x)-\frac{x f(x)}{x x} x .
$$

Since $x g(x)=x f(x)-x f(x)=0$, then the function $g$ meets Walras' law. Let us fix a number $h>0$ and define a function $z^{h}:$ int $\Delta^{n} \rightarrow \mathbb{R}^{n}$ as

$$
z^{h}(x)=\left(z_{1}^{h}(x), \ldots, z_{n}^{h}(x)\right):=\left(g_{1}(x)+h\left(\left(n x_{1}\right)^{-1}-1\right), \ldots, g_{n}(x)+h\left(\left(n x_{n}\right)^{-1}-1\right)\right) .
$$

One can easily check that $z^{h}$ is an excess demand function. Now, by the corollary, we see that for each $h>0$ there exists a point $x^{h} \in \operatorname{int} \Delta^{n}: z^{h}\left(x^{h}\right)=0$, written equivalently as

$$
g_{i}\left(x^{h}\right)=-h\left(\frac{1}{n x_{i}^{h}}-1\right), \quad i \in[n] .
$$

Let $h \rightarrow 0^{+}$and $x^{h} \rightarrow x \in \Delta^{n}$ (taking a subsequence if necessary). If $x_{i}>0$, then $g_{i}(x)=0$. If $x_{i}=0$ then $\frac{1}{n x_{i}^{h}} \rightarrow+\infty$ and $\frac{1}{n x_{i}^{h}}-1 \rightarrow+\infty$, so $-h\left(\frac{1}{n x_{i}^{h}}-1\right)<0$, but boundedness of $g$ implies that $-h\left(\frac{1}{n x_{i}^{h}}-1\right), h>0$, is bounded. We obtain $g(x) \leq 0$, which ensures that $f(x)=x$ (see [7]). Hence, to find an approximate fixed point of $f$, we can apply the algorithm for $z^{h}, h$ sufficiently small.

The equivalence of the existence of equilibria for excess demand functions defined on the standard closed simplices ${ }^{1}$ and Brouwer's theorem was shown in [7]. The proofs of the equivalence for the excess demand functions considered in the current paper can be found in [8] or [9].

\subsection{Open questions}

Combinatorial Lemma 1 seems to be interesting for its own sake in spite of the fact that it is proved for a particular triangulation. We have seen that it implies the existence of equilibrium for an excess demand function. A slight modification of the proof of Theorem 7 in [8] allows to claim that the existence of equilibrium for an excess demand function is equivalent to the Brouwer fixed point theorem (see also [9]). The famous Sperner lemma, which is a combinatorial tool used to prove Brouwer's fixed point theorem (and which is equivalent to it [4, p.21]) has many implications (e.g., see [10, pp.101-103]). What are other implications of Lemma 1? Does Lemma 1 generalize to any triangulation of the standard simplex? Is it equivalent to Sperner's lemma? What about the behavior of the algorithm presented in the paper in comparison to the behavior of other computational methods for finding equilibria (e.g., methods presented in [4])? How to modify the algorithm to allow for the computation of (approximate) equilibria of excess demand mappings rather than functions? 


\section{Acknowledgements}

I would like to thank participants of the Seminar of Department of Mathematical Economics (Poznań University of Economics), Nonlinear Analysis Seminar at Faculty of Mathematics and Computer Science (Adam Mickiewicz University in Poznań), Seminar of the Game and Decision Theory at Institute of Computer Science (Polish Academy of Science, Warsaw) for helpful comments and criticism. I also thank the referees for their comments and remarks that improved the paper. All remaining errors are mine. This work was financially supported by the Polish National Science Centre, grant no. UMO-2011/01/B/HS4/02219.

\section{Endnotes}

a Precise definitions can be found in [1] or [2, Chapter 1]. The presented description of exchange economies goes along the lines of [2, pp.29-31] and is rather standard.

b Homogeneity of degree zero is among these conditions: we can restrict our considerations to excess demand functions defined on the open standard simplex and not on the whole positive orthant of $\mathbb{R}^{n}$ - see Definition 1 in Section 4.

c Constructive in the sense that it allows to derive a (simplicial) algorithm for computation of an approximate equilibrium.

d We find [4] by Yang as a comprehensive source of information on computation of equilibria and fixed points. Since the primary goal of this paper is to derive the existence of equilibria in a novel way without referring to Brouwer's fixed point theorem and not to construct algorithm for computation of equilibria, the algorithm presented below should be treated as a by-product which is important, as we believe, but whose properties should be examined in the future.

e Our K-triangulation is called the $K_{2}(m)$-triangulation in [4, p.64].

$f$ We could not have found a reference for this statement but it is proof is elementary.

9 For simplicity: if we know that $\sigma$ is a simplex we write $/(\sigma)$ instead of - formally correct way - $(V(\sigma))$. Notice, that the codomain of the function / could be easily changed to $[n-1]$ in place of $[n] \cup\{0\}$, but we do not do that to discern the 'top' of a simplex from its 'bottom' - see Figure 1.

$\mathrm{h}$ The method of construction of the sequence is similar to the one used in the proof of the correctness of the Scarf algorithm - see $[4$, p.68].

i The idea for the definition of $\tilde{z}$ comes from the proof of Theorem 1 in [6] as it comes as a loose suggestion for the proof of our main theorem below.

j The idea for / is closely related to the notion of the standard integer labeling rule [4, p.63].

k See [2, Theorem 1.4.4] or [1, Lemma 4].

I This assumption eliminates both boundary conditions presented above.

\section{Received: 11 January 2013 Accepted: 5 April 2013 Published: 18 April 2013}

\section{References}

1. Debreu, G: Existence of competitive equilibrium. In: Arrow, KJ, Intriligator, MD (eds.) Handbook of Mathematical Economics, vol. 2, pp. 697-743. North-Holland, Amsterdam (1982)

2. Aliprantis, C, Brown, D, Burkinshaw, O: Existence and Optimality of Competitive Equilibria. Springer, Berlin (1990)

3. Mas-Colell, A: On the equilibrium price set of an exchange economy. J. Math. Econ. 4, 117-126 (1977)

4. Yang, Z: Computing Equilibria and Fixed Points. Kluwer, Boston (1999)

5. Scarf, H: The computation of equilibrium prices: an exposition. In: Arrow, KJ, Intriligator, MD (eds.) Handbook of Mathematical Economics, vol. 2, pp. 1006-1061. North-Holland, Amsterdam (1982)

6. Maćkowiak, P: The existence of equilibrium without fixed-point arguments. J. Math. Econ. 46(6), 1194-1199 (2010)

7. Uzawa, H: Walras' existence theorem and Brouwer's fixed-point theorem. Econ. Stud. Q. 13(1), 59-62 (1962)

8. Maćkowiak, P: Some equivalents of Brouwer's fixed point theorem and the existence of economic equilibrium. In: Matłoka, M (ed.) Quantitative Methods in Economics. Scientific Books, vol. 222, pp. 164-171. Poznań University of Economics Press, Poznań (2012)

9. Toda, M: Approximation of excess demand on the boundary and equilibrium price set. Adv. Math. Econ. 9, 99-107 (2006)

10. Dugundji, J, Granas, A: Fixed Point Theory. Springer, New York (2003)

doi:10.1186/1687-1812-2013-104

Cite this article as: Maćkowiak: The existence of equilibrium in a simple exchange model. Fixed Point Theory and Applications 2013 2013:104. 